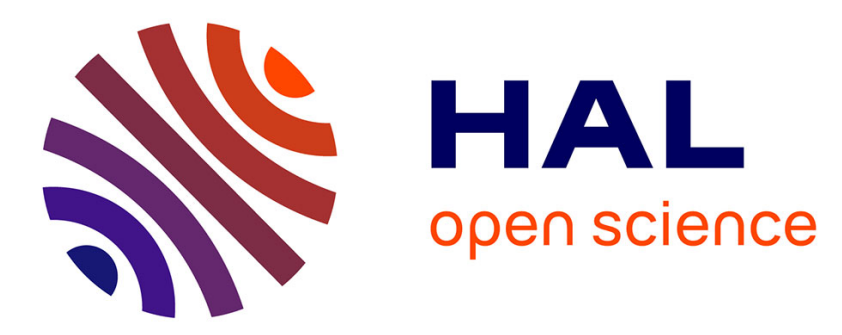

\title{
Direct Comparison of a Capacitive to an Electro-Optic Electric Field Sensor
}

\author{
Didier Robbes, Achille Junior Mbogol Touye, Corentin Jorel, Gwenaël \\ Gaborit, Lionel Duvillaret
}

\section{- To cite this version:}

Didier Robbes, Achille Junior Mbogol Touye, Corentin Jorel, Gwenaël Gaborit, Lionel Duvillaret. Direct Comparison of a Capacitive to an Electro-Optic Electric Field Sensor. 12th International Conference On Sensing Technology (ICST 2018), Dec 2018, Limerick, Ireland. 10.1109/ICSensT.2018.8603614 . hal-01989869

\section{HAL Id: hal-01989869 https://hal.science/hal-01989869}

Submitted on 22 Jan 2019

HAL is a multi-disciplinary open access archive for the deposit and dissemination of scientific research documents, whether they are published or not. The documents may come from teaching and research institutions in France or abroad, or from public or private research centers.
L'archive ouverte pluridisciplinaire HAL, est destinée au dépôt et à la diffusion de documents scientifiques de niveau recherche, publiés ou non, émanant des établissements d'enseignement et de recherche français ou étrangers, des laboratoires publics ou privés. 


\section{Direct Comparison of a Capacitive to an Electro-Optic Electric Field Sensor}

\author{
$1^{\text {st }}$ Didier Robbes \\ GREYC \\ UNICAEN,ENSICAEN and CNRS \\ Caen, France \\ didier.robbes@unicaen.fr
}

\author{
$2^{\text {nd }}$ Mbogol Touye Achille Junior \\ GREYC \\ UNICAEN,ENSICAEN and CNRS \\ Caen, France \\ achille-junior.mbogol-touye@ unicaen.fr
}

\author{
$3^{\text {rd }}$ Corentin Jorel \\ GREYC \\ UNICAEN,ENSICAEN and CNRS \\ Caen, France \\ corentin.jorel@unicaen.fr
}

\author{
$4^{\text {th }}$ Gwenaël GABORIT \\ Kapteos S.A.S. \\ St Hélène-du-Lac, France \\ gwenael.gaborit@kapteos.com
}

\author{
$5^{\text {th }}$ Lionel Duvillaret \\ Kapteos S.A.S. \\ St Hélène-du-Lac, France \\ lionel.duvillaret@kapteos.com
}

\begin{abstract}
A Sensor to measure electric vector fields based on the Pockel effect and a sensor making good use of a search capacitor have been submitted to the same electric field excitations. Outputs of their analog electronics are further processed using a multi-channel dynamic signal analyzer $(24$ bits and $10 \mathrm{mHz}$ to $100 \mathrm{kHz}$ analysis bandwidth). Both AC and DC behavior have been addressed, the later with the help of an electric field mill. The direct comparison was made possible up to $100 \mathrm{kVm}^{-1}$ using a large enough electric field source and high voltage outputs amplifiers. This way, the sensors are operated at the same time, while occupying similar positions inside the electric field. Results expressed as a voltage transfer function in the AC mode exhibit a good coherence all along the analysis band. It highlights the different bandwidth of the two instruments nonetheless sharing three decades: the search capacitor working at low frequency while the electro-optic one is known to work at high frequency. Finally, the electric field mill used at a chopping frequency of $54 \mathrm{~Hz}$ induces a limitation for both sensors in the lower part of their measuring range, below a few hundreds of $\mathrm{Vm}^{-1}$, while noise floors of each sensor are found well below a residual signal induced by the electric field mill rotation.
\end{abstract}

Index Terms-Electric field sensors, DC, directivity, field mill

\section{INTRODUCTION}

History of the classical electromagnetism theory together with its numerous applications may reveal some unexpected features. It appears that measuring instruments dedicated to magnetic field are multitude, while those dedicated to electric field are not, more especially in the very low frequency domain, starting from DC. Alternatively, in the ultra-high frequency domain, although the situation is somewhat different, due to the direct link between the magnetic and the electric fields through the wave impedance, measure of electric field strength, when approaching the disruptive limit, forbids the use of any conducting material in the sensor design, even a few tens of meters apart from the sensor active volume. The classical approach to sense electric field in the low frequency range is based on the use of electrostatic influence upon a highly conducting electrode, or capture electrode, in association with a system of auxiliary electrodes, or electric field mill, that move around the first one [1].The capturing electrode alone is coupled either to an electrometer type amplifier that exhibits an ultra-high input impedance ( $1 \mathrm{G} \Omega$ or more) or to a charge amplifier. In the commercially available configurations of such electrometers, only one capture electrode is used to sense the chopped voltage signal produced by a far charge distributions and only an evaluation of the electric field strength is obtained, with no good nor well-characterized directivity [2] .

In this paper, we directly compare two electric field sensors, the behavior of which based on two complementary measuring principles. One is based on electrostatic influence upon free charge at the surface of highly conducting metals. The other is based on the Pockel effect : the electric field modifies the electronic clouds of atoms inside an optically active material, such as the Bismuth Silicon Oxide (BSO).

The first measuring principle is operated using a gradiometric arrangement of a pair of capturing electrodes, or search capacitor. As usual in gradiometric techniques, the output is an estimation of a gradient component of the input physical quantity, here the space-time voltage variations.

The other method is not a gradiometric one: the electric field action, through the Coulomb force, is volumetric and at the scale of an optically active crystal. Induced effects are probed by the means of coherent light conveyed through dielectric guides, which allows an entire sensing system without conducting parts. This of course, is not possible with search capacitor. Besides these complementarity in measuring principles and physical implementation, one is highly sensitive down to the low frequency range, while the other has a very low sensitivity, but with extended capabilities both for very high values of the field strength and in the ultra-high frequency range. Because the dynamic range of each technologies is large, and because there is a common part of their frequency range, between $10 \mathrm{~Hz}$ to $100 \mathrm{kHz}$, a direct comparison was done, the results of which are presented here. The paper is arranged as follows. In part II, we present the experimental 


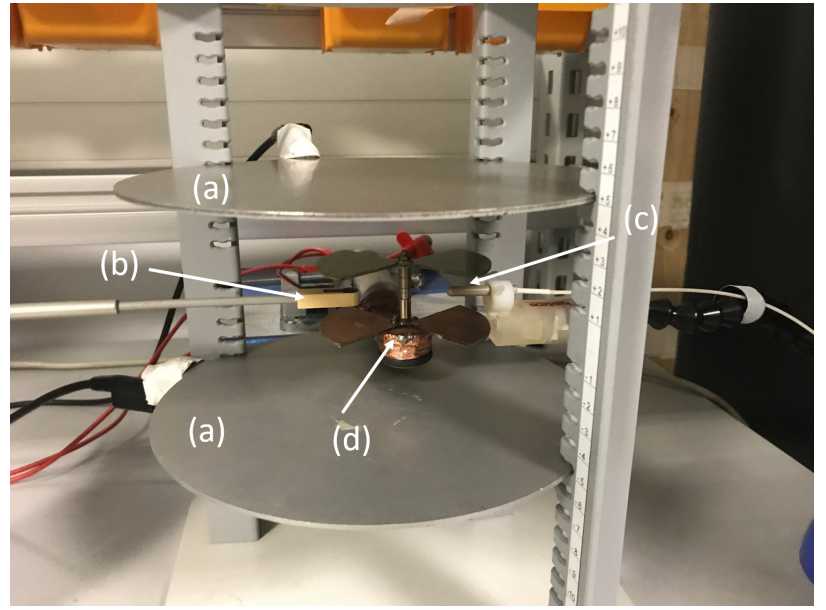

Fig. 1. Photography of the experimental set-up. (a) Conducting disks for field sourcing. (b) Search Capacitor Gradiometer. (c) Electro-Optic Probe. (d) Butterfly field mill and its motor.

setup and the sensors. Measurements are discussed in part III, while perspectives and conclusion are presented in part IV.

\section{EXPERIMENTAL SET-UP}

\section{A. Field sourcing}

Dedicated instrumentation to create high quality electric fields are neither fully commercialized nor a subject of R\&D. Many reasons can be appealed to appreciate the above assumption. High and low frequency domains, as separated by the wave behavior of electromagnetic fields, are not covered by the same means of sourcing. While the first is easily provided by the means of various antennas, the other is also easily obtained using a set of conductors separated by a space gap, where the electric field is developed, both as a function of the voltage difference and of the distance in between the conductors. Both domains suffer from some drawbacks. One is inherent to the presence of a magnetic field in the high frequency range, while operation at high field in the low frequency range is made difficult when a large volume with a good spatial homogeneity of the enclosed field is needed. In order to be able to compare directly the electro-optic (EO) Probe with the search capacitor gradiometer (SCG), a sourcing device able to deliver an electric field as large as $100 \mathrm{kVm}^{-1}$ had to be used, because of their great difference in sensitivity.

As shown in the picture Fig. 1 of the set up, we use two 20$\mathrm{cm}$-diameter conducting disks biased with two voltage sources, which are antisymmetric relatively to the reference voltage. Disk axis is along the $\mathrm{z}$ direction; and the distance between the plates is adjustable from 2 to $20 \mathrm{~cm}$, by $1 \mathrm{~cm}$ steps. In DC mode, top and bottom electrodes must have opposite voltage values with respect to their common reference. In the same way, in $\mathrm{AC}$ mode, one voltage is $180^{\circ}$ phase shifted relatively to the other. This allows having the middle symmetry plane between the disks that stays at the reference level at any time. Two high voltage amplifiers Trek Model 2220 with fixed gain of 200 are used to generate high voltage signals within the range +/- $2000 \mathrm{~V}$, while the output current is limited to a maximal value of $10 \mathrm{~mA}$. It follows that an electric field with a maximum strength is obtained using $\mathrm{a}+/-10 \mathrm{~V}$ input voltage from a standard function generator and a unity gain voltage inverter. Furthermore, the frequency range, investigated up to $100 \mathrm{kHz}$, is limited by the bandwidth of the amplifiers, the latter only slightly dependent on the output amplitude when operating in the large signal limit. This is visible in Fig. 2 where transfer functions of the two electrometers are plotted.

\section{B. Sensors}

Two sensors are characterized in this paper. The electrooptic (EO) probe developed by Kapteos S.A.S (eoSense instrument) [3] and a search capacitor gradiometer (SCG) based around a differential charge amplifier as described in patent [4]. Both sensors can be seen on Fig. 1.

The fully dielectric and millimeter sized optical Probe is based on the Pockels effect, also called EO effect. This pigtailed Probe involves a non-centrosymmetric crystal (BSO), which sees its Eigen refractive index linearly modified by the E-field. The transduction is vectorial meaning that each Eigen component of the E-field vector can be measured in real time. The intrinsic bandwidth of the Probe covers more than 8 decades of frequency from $30 \mathrm{~Hz}$ up to several $\mathrm{GHz}$. The achievable dynamic range of the E-field measurement exceeds $130 \mathrm{~dB}$ per unit bandwidth, with a minimum measurable field lower than $1 \mathrm{~V} / \mathrm{m}$ and a compression point greater than 1 $\mathrm{MV} / \mathrm{m}$. The spatial resolution of the EO Probe is weaker than $1 \mathrm{~mm}^{3}[3]$.

The SCG electrometer is build around a pair of millimeter sized and circular electrodes made of copper and separated by a base line of $5 \mathrm{~mm}$. Evaluation of the $\mathrm{z}$ component of the voltage gradient is obtained together with the mean voltage variation in the middle of that base line. This is obtained by the means of a differential charge amplifier subjected to a specific feedback process, as depicted in [4]. The conditioning unit outputs two signal, one that scale with the time voltage variation having the in-band transfer parameter of $0,1 \mathrm{~V} / \mathrm{V}$, while the second one scales with the voltage gradient, with its in-band transfer parameter very close to $1 \mathrm{mV} /\left(\mathrm{Vm}^{-1}\right)$.

\section{Field mill}

The field mill (see photo Fig. 1) is a double butterfly shutter made with standard FR4 PCB boards. It is biased at the reference voltage that alternatively shields and expose the sensors which are symmetrically placed just in the middle of the mill wings. It follows that the electric field sensed by the EO Probe and the SCG no longer is at DC, but the amplitude seen at twice the rotation frequency scales with the DC field value. That simple field mill is just powered by a DC voltage, in the range of 5 to $15 \mathrm{~V}$, and was operated at a value of $8 \mathrm{~V}$, to get about 27 rotations per second, which means a chopping frequency of $54 \mathrm{~Hz}$.

\section{Monitoring}

A dynamic signal analyzer (CF-9400 from Ono Sokki) has been used to monitor signals associated to the direct 


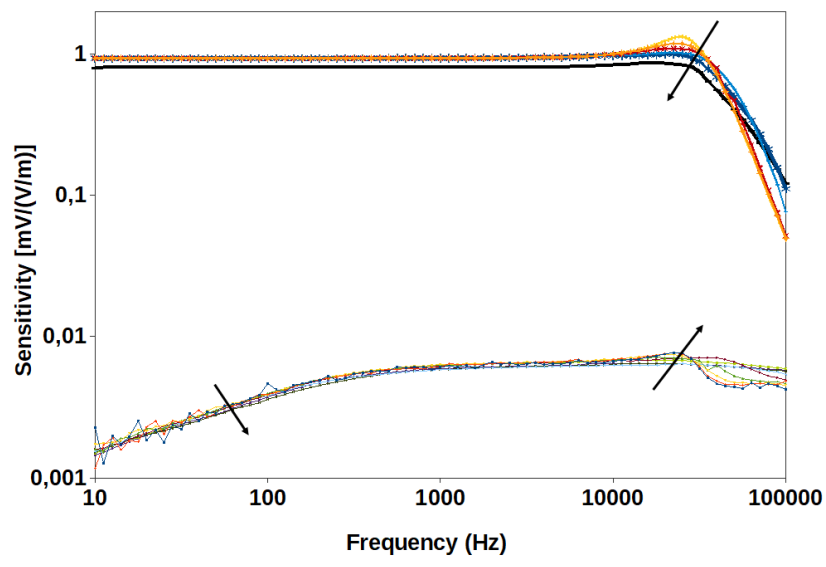

Fig. 2. Transfer function of both sensor sensitivty (SCG above EO probe). SCG has been characterized at fields increasing from $200 \mathrm{~V} / \mathrm{m}$ to $8 \mathrm{kV} / \mathrm{m}$. EO probe has been characterized at fields increasing from $200 \mathrm{~V} / \mathrm{m}$ to $40 \mathrm{kV} / \mathrm{m}$ with doubling steps. Arrows indicate the increasing field direction.

comparison of the EO an SCG sensors. It has a capability to operate in the $10 \mathrm{mHz}$ to $100 \mathrm{kHz}$ bandwidth with up to 4 analog input channels with 24 bits AD converters. Transfer functions monitoring together with most of the modern signal analysis are performed in real time, which greatly helps the direct comparison.

\section{RESULTS}

\section{A. AC field transfer functions}

In that subsection, we report on the $\mathrm{AC}$ measurements of both transfer functions, in the $10 \mathrm{~Hz}$ to $100 \mathrm{kHz}$ range as shown on Fig. 2. Amplitude of the sinusoidal and antisymmetric voltage excitation of the E-field sourcing device (subsection $2 \mathrm{~A}$ ) was varied between $10 \mathrm{~V}$ to $2000 \mathrm{~V}$. Using the signal analyzer, direct comparison was made in real time, while varying the frequency in the specified range. Plots of Fig. 2 show two families of the amplitude Bode diagrams, associated to each value of the field excitation amplitude. Features shown here are :

- The great difference in values of the in-band sensitivity : around $6.4 \mu \mathrm{V} /\left(\mathrm{Vm}^{-1}\right)$ for the EO device and $930 \mu \mathrm{V} /\left(\mathrm{Vm}^{-1}\right)$ for the SCG device.

- A slight difference in the dispersion of the in-band transfer values around its mean: $7.7 \%$ for the EO device and $0.04 \%$ for the SCG device.

- A common high frequency perturbation $(20-100 \mathrm{kHz})$, due to the upper limit of the amplifier's transfers that were not corrected during the real time data acquisition and direct monitoring.

- A low frequency cut-off of the EO device, both due to the crystal defects and to the conditioning unit. This eliminates parasitic low frequency signals due to vibration and temperature effects in the long laser fiber that couples the Probe to the conditioning unit. The cut off is below 200 $\mathrm{Hz}$ at $8 \mathrm{~dB} / \mathrm{Dec}$. The low frequency cut off the SCG device at $0.1 \mathrm{~Hz}$ is not seen, because it is almost three decade below

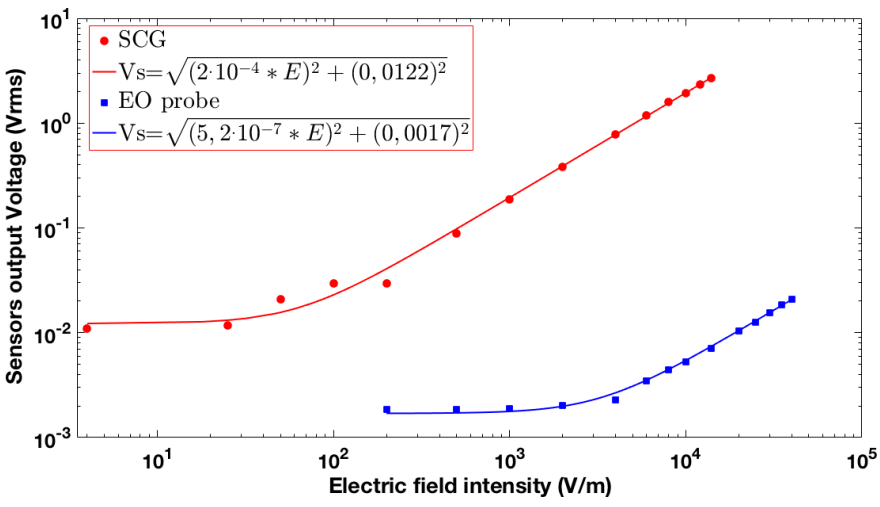

Fig. 3. Sensors output voltage (VRMS) vs. DC Electric Field. (red, up) SCG. (Blue, below) EO probe. Lines nonlinear fit using the relation explicited in the text box.

the lower limit of the excitation in this set of experiments (10 $\mathrm{Hz}$ ). In addition, a more detailed examination of the in-band.

- Transfer in the range $500 \mathrm{~Hz}$ to $10 \mathrm{kHz}$ shows a very flat behavior of the SCG while the EO probe is not, exhibiting a small slope of about $0,7 \mathrm{~dB} / \mathrm{dec}$. This behavior has not been previously identified, nor its origin previously discussed.

- A sensitivity loss for the SCG transfer obtained at the higher field of $8000 \mathrm{Vm}^{-1}$ (black line below $1 \mathrm{mV} /\left(\mathrm{Vm}^{-1}\right)$ on fig2). It is attributed to the limited range of voltage output of its conditioning unit. Once the higher limit is reached, classical and strong signal distortion appears which lead to artifacts in the measured transfer.

\section{B. DC field measurements with the field mill}

As depicted subsection II.C, the two sensors have active volumes in the millimeter range with either a conducting access or a dielectric access in a direction transverse to the $\mathrm{z}$ field direction. Due to the mill conception with two butterfly wings, both devices could probe quasi-symmetric AC signal as modulated by the rotating mill. The real time FFT computation and monitoring of the output signals was then simultaneously available, again greatly facilitating the direct comparison of the two sensors, which are now able to estimate the DC component of the applied field. Outputs of the monitoring are the RMS value of the probes outputs at the $54 \mathrm{~Hz}$ frequency line, twice the rotation frequency of the mill. Because less than $50 \%$ of the applied field reaches the probes placed inside the mill, the DC maximum measured field reached the value of 14 $\mathrm{kVm}^{-1}$ for the SCG (distortion limit) and $80 \mathrm{kVm}^{-1}$ for the EO Probe (sourcing plate's spacing limit). Results are shown on Fig. 3, which plots RMS values of the $54 \mathrm{~Hz}$ lines, versus the applied DC field. From that plots, in their linear part, roughly above $100-1000 \mathrm{Vm}^{-1}$, one extracts sensitivities values : $283 \mu \mathrm{V} /\left(\mathrm{Vm}^{-1}\right)$ for the SCG and $0.73 \mu \mathrm{V} /\left(\mathrm{Vm}^{-1}\right)$ for the EO probe. Values are obtained from the slope in Fig. 3 corrected by $\sqrt{2}$. This allows to evaluate the proper value of the field mill extra attenuation. Having in mind an ideal chopper of the DC signal, the latter should be seen by the sensors as a square signal, due to the shape of the copper covered part of 


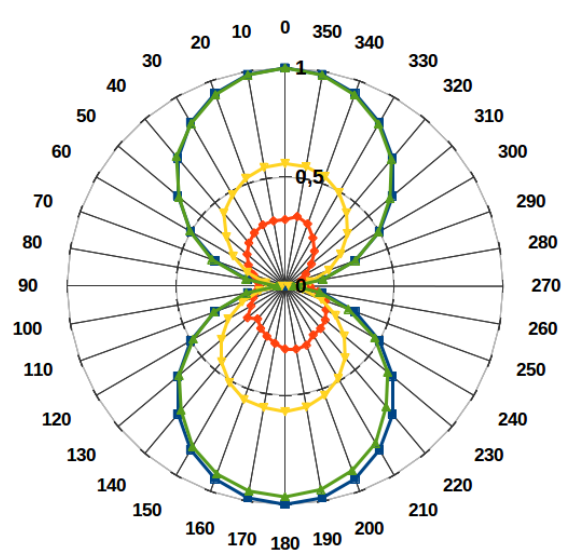

Fig. 4. Normalized sensors output voltage (VRMS) vs sensor direction $\vec{n}$ relative to the vertical direction $\overrightarrow{u_{z}}\left(0^{\circ}\right)$ of the AC electric field $(200 \mathrm{~V} / \mathrm{m})$. (Blue) Cosine guildeline. (Green) SCG alone used for normalizing. (Yellow) With the field mill in on position. (Orange) With the field mill in off position. The latter values are mutiplied by an order of magnitude to be seen.

the wings versus the identical shape of the uncovered one. In such an ideal chopper, only half of the signal is transmitted to the measuring system. It follows that the ideal DC transfer through the chopper should be half of that in the ac mode, meaning for example $465 \mu \mathrm{V} /\left(\mathrm{Vm}^{-1}\right)$ for the SCG in the field mill. Because we observe only $283 \mu \mathrm{V} /\left(\mathrm{Vm}^{-1}\right)$, this implies our field mill exhibits some extra attenuation which limits the transmission of the available field to about $59 \%$ of the ideal value. As it will be made clear in the next subsection, the efficiency of the field mill is related to the ratio of the exposed to shielded field during half of a turn of the pair of wings.

Both plots of DC transfer exhibit a deviation to the ideal behavior at low field, because a flat part in the curves is clearly seen in fig. 3. It is associated to the mill's rotation, and could, at first sight, be associated to a parasitic field from the mill itself. From the asymptotic values of the voltage outputs at low field, together with values of the transfers at higher fields, one could expect to identify a possible input offset error, but the interpretation is not so straightforward. First of all, one cannot neglect possible phase effects, meaning that phase differences and not only amplitude ratio should be taken into account, especially if the parasitic field associated to the rotating mill alone is probably not as homogeneous as the one to be measured. Additionally, more careful experiments should be done, but are made difficult at low values of the fields, especially for the EO probe, where noise limits are reached.

\section{Directivity}

Having a look on commercially available AC electrometers, most of them are only displaying magnitude of the electric field, but not its direction. This is also the case in DC for the commercially available electric field mills. It follows that characterization of directionality of the EO and SCG electrometers is mandatory, for both AC and DC operating modes. Careful directivity characterizations are presented here for the

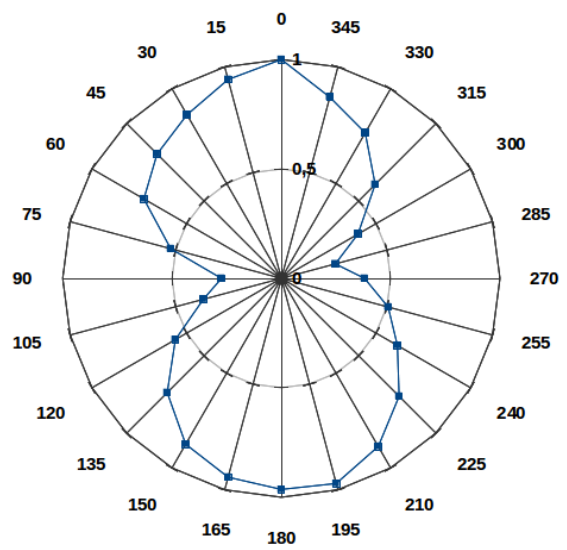

Fig. 5. Normalized sensors output voltage (VRMS) vs SCG and field mill direction $\vec{n}$ relative to the vertical direction $\overrightarrow{u_{z}}\left(0^{\circ}\right)$ of the DC electric field $(200 \mathrm{~V} / \mathrm{m})$

SCG system alone and inside the electric field mill. The later configuration allows getting a better characterization of the mill. As a matter of fact, the modulated field inside the mill, is measured by the included directional sensor, the response of which must, in some way, be sensitive to the original field direction. Fig. 4 report directionality plots obtained in three different configurations.

The reference configuration is provided by the SCG alone, at the in-band frequency of $1 \mathrm{kHz}$. The gradiometric arrangement of the SCG has the normal direction $\vec{n}$ of its capturing electrodes. Introducing the unit vector $\overrightarrow{u_{z}}$ in the $\mathrm{z}$ direction of the applied electric field, the SCG response scales with the scalar product $\vec{n} \cdot \overrightarrow{u_{z}}$ It follows almost superimposed on the cosine function. This standard behavior is clearly marked upon the external plot reported Fig. 4, and shows the excellent directivity of the SCG.

The two other configurations include the electric field mill and the SCG device, but in two static positions: one corresponds to the "ON" state of the chopper that exposes the SCG to the field, while the second corresponds to the "OFF" state, where the SCG should be fully shielded. From these plots, characteristics of the "ON" state can be extracted : the exposed field keeps a quasi-constant direction, while its amplitude, in the direction of the maximum of the directivity pattern (nulle angle), is around $-5 \mathrm{~dB}$ below the applied field. Alternatively, in the "OFF" state, the shielding is not fully complete: it is of about $-25 \mathrm{~dB}$ below the value in the "ON" state. To link that work to the one of the precedent paragraph, notice that the linear value corresponding to the $-5 \mathrm{~dB}$ attenuation in the "ON" state, $56 \%$, is close to that found from the ratio of the $\mathrm{DC}$ and $\mathrm{AC}$ transfer at the rotating frequency of the mill, 59\%, which comfort the coherence of our analysis.

A fourth work has been carried out on the directivity of the field mill measuring process, which, to our knowledge has not been presented before. In that part, both the SCG and the rotating field mill are operated for a set of $15^{\circ}$ 
angle steps. Results are reported on Fig. 5. It shows that the directivity pattern still presents a preferential direction, but not as pronounced as the one of the SCG alone.

\section{PERSPECTIVES AND CONCLUSION}

A direct comparison between two electric field sensors based on complementary physical working principles has been presented. This was made possible due to a common band in their working range in the frequency domain. The use of a real time dynamic signal analyzer having a high dynamic range also greatly facilitated that direct comparison, despite the large difference in the respective sensitivities of the sensors. The use of two high voltage output amplifiers, together with the combination of a symmetric set of large conducting plates fed by antisymmetric voltages allowed the operation of both sensors at the same time, while they were subjected to the same electric field excitation. Because none of the sensors operates at DC, the use of an electric field mill make it possible, keeping in the purpose of the direct comparison, a field mill was conceived to operate both sensors in a common chopped DC field. We found a good consistency for both technologies in the AC mode except, for the small slope $(0,7$ $\mathrm{dB} / \mathrm{dec}$, in-band) in the transfer function of the $\mathrm{EO}$ probe. In the DC mode, at high fields above a few $\mathrm{kV} /\left(\mathrm{Vm}^{-1}\right)$, a good consistency is also found. At low DC field values, a discrepancy was noticed from the Fig. 3 examination, meaning that operating electric field mills at low fields may reveal some drawbacks. The latter have to be understood and circumvented if one still wants to use them as reference to accurately measure DC electric fields. At DC, other approaches that are known, such using MEMs devices [5],[6]or only using rotation of the SCG itself as in [4], could also find benefits from the direct comparative method that was used here. This could help to get a good state of the art of sensing technologies dedicated to electric field, static, quasi static or even at much higher frequencies. Because in our mind, such a state of the art is missing, ways to improve it will need better high field sourcing devices, and DC sensing technologies operated with no moving parts. When aiming for a large band (DC-MHz) sensor, both sensor used here with a field mill have strong limitations.

\section{REFERENCES}

[1] D. M. Taylor and P.E. Secker, Industrial Electrostatics: Fundamentals and Measurements, New York, Research studies Press, Wiley, 1994.

[2] H. Bloemink, "Static electricity measurements for lightning warnings; an exploration", De Bilt 2013 I Internal report, pp. 2013-01.

[3] G. Gaborit et al., "Single Shot and Vectorial Characterization of Intense Electric Field in Various Environments With Pigtailed Electrooptic Probe," in IEEE Transactions on Plasma Science, vol. 42, no. 5, pp. 1265-1273, May 2014.

[4] D. Robbes, "Physical Quantity Measuring Unit and device for Measuring a Voltage and an Electric Field", European Patent Application No. 09290213.9, $20^{\text {th }}$ March 2009.

[5] T. Chen, C. Shafai, A. Rajapakse, B.Y. Park, Micromachined Electric Field Mill Employing a Vertical Moving Shutter, Procedia Engineering, Volume 87, 2014,Pages 452-455.

[6] S. Ghionea, G. Smith, J. Pulskamp, S. Bedair, C. Meyer and D. Hull, "MEMS electric-field sensor with lead zirconate titanate (PZT)-actuated electrodes," 2013 IEEE SENSORS, Baltimore, MD, 2013, pp. 1-4. 